\title{
ANÁLISE DOS PADRÕES DE INVESTIMENTOS DOS HOSPITAIS BRASILEIROS COM BASE NA ANÁLISE ENVOLTÓRIA DE DADOS (DEA)
}

\author{
Data de submissão: 03/03/2014 \\ Aceite: 30/05/2016 \\ Antônio Artur de Souza ${ }^{1}$ \\ Ewerton Alex Avelar ${ }^{2}$ \\ Amanda Lucchesi Lara ${ }^{3}$ \\ Marcella Rios Gonçalves ${ }^{4}$ \\ César Urbano de Souza ${ }^{5}$
}

\section{RESUMO}

Este artigo apresenta os resultados de uma pesquisa que visou identificar padrões ótimos de investimentos para hospitais brasileiros entre os anos de 2006 e 2011. Os dados operacionais utilizados foram coletados no Banco de Dados do Sistema Único de Saúde (DATASUS) e os dados financeiros foram obtidos por meio das demonstrações contábeis disponíveis via Internet. Para a análise dos dados, foram empregadas diferentes técnicas: estatística descritiva, teste de Kruskal-Wallis, Coeficiente de Correlação de Spearman e a análise envoltória de dados (Data Envelopment Analysis - DEA). Constatou-se que a natureza jurídica e a unidade da federação de origem influenciaram a eficiência dos hospitais entre os anos de 2006 e 2011. Os resultados demonstram que os hospitais sem fins lucrativos se esforçam para atender a um maior volume de pessoas em suas unidades, enquanto as organizações com fins lucrativos empregam os seus recursos com forte orientação a resultados financeiros. Verificou-se que cinco hospitais apresentaram padrões de investimentos que podem ser considerados ótimos segundo os modelos desenvolvidos.

Palavras-chave: Hospitais. DEA. Investimentos.

\footnotetext{
1 Possui graduação em Ciências Contábeis pela Universidade Federal de Santa Catarina, UFSC, mestrado em Engenharia de Produção pela UFSC e doutorado em Management Science pela The University of Lancaster, LANCS UNIV, Grã-Bretanha. Belo Horizonte - MG. Brasil. E-mail: antonioarturdesouza@gmail.com

2 Possui graduação em Ciências Contábeis pela Universidade Federal de Minas Gerais, UFMG, mestrado em Administração pela Universidade Federal de Lavras, UFLA e doutorado em Administração pela UFMG. Belo Horizonte - MG. Brasil.E-mail: alexavelar2003@ yahoo.com.br

3 Possui graduação em Ciências Atuariais pela Universidade Federal de Minas Gerais, UFMG. E-mail: nandexll@hotmail.com 4 Graduação em andamento em Ciências Contábeis pela Universidade Federal de Minas Gerais, UFMG. E-mail: marcellarios2004@ yahoo.com.br

5 Possui graduação em Direito pela Faculdades Milton Campos e graduação em andamento em Administração pela Universidade Federal de Minas Gerais, UFMG. E-mail: urbanocesar@gmail.com
} 


\section{INTRODUÇÃO}

Para Ozgulbas e Koyuncugil (2009), o aprimoramento dos serviços de saúde é uma questão essencial para as políticas públicas. Nesse sentido, autores como Guerra (2011) destacam que os hospitais são considerados essenciais na prestação de serviços de saúde. Apesar da sua importância, aspectos ligados ao gerenciamento de estoques para atender as necessidades dos pacientes colocam a prestação do serviço médico-hospitalar na pauta das atividades mais complexas no mercado (MEDEIROS et al., 2009). De acordo com Struett (2005), os hospitais são organizações intricadas fornecedoras de multiprodutos, com vários processos de produção altamente interdependentes, cujos resultados se articulam para o fornecimento de seu principal produto: o diagnóstico e o tratamento do paciente.

A gama de serviços hospitalares exige atenção redobrada por parte dos gestores a fim de garantir a qualidade dos serviços prestados. Assim, são necessárias práticas de controle que permitam detectar com antecedência as situações que possam gerar consequências aos pacientes e familiares, e à organização como um todo (NOVARETTI, 2014). Este gerenciamento dos riscos nos procedimentos hospitalares e a prevenção de eventos adversos não dizem respeito apenas aos funcionários de saúde, mas também aos gestores das organizações (NOVARETTI, 2014). Dessa forma, é importante criar políticas para a melhoria da gestão hospitalar. Todavia, o que se verifica é que os hospitais brasileiros usualmente enfrentam graves problemas de gestão (SOUZA et al., 2009; GUERRA et al., 2012). Ressalta-se, ainda, a extensão continental do país, que implica em uma série de peculiaridades regionais que influenciam também na gestão hospitalar no Brasil (O'DWYER et al., 2013). Ademais, características específicas de cada hospital, tais como o porte e a natureza jurídica, também contribuem para isso (VELOSO, MALIK, 2010).

Ressalta-se que um aspecto essencial da gestão hospitalar está relacionado à gestão financeira (GRUEN; HOWARTH, 2005). Diversos estudos veem destacando a importância da administração dessa área nos hospitais, com ênfase no emprego de indicadores financeiros, com a finalidade de analisar o desempenho destes (ZELLER et al., 1996; BITTAR, 2001; OZGULBAS; KOYUNCUGIL, 2009; VELOSO, MALIK, 2010).

Ademais, Ancarani et al. (2009) destacam a importância da análise de eficiência nos serviços hospitalares, enquanto Guerra et al. (2012) ressaltam o emprego de técnicas como a Análise Envoltória de Dados (Data Envelopment Analysis - DEA) na avaliação da eficiência hospitalar. Apesar da importância do tema, tanto em aspectos teóricos como práticos, a gestão dessas organizações, especialmente no que se refere à análise de eficiência, é pouco abordada na literatura (ANCARANI et al., 2009). Nesse sentido, embora seja o componente predominante do sistema de saúde no Brasil, a gestão de hospitais até recentemente vinha recebendo pouca atenção, tanto dos formuladores de políticas como dos pesquisadores (GUERRA, 2011).

Nesse sentido, este artigo apresenta os resultados de uma pesquisa que visou identificar padrões ótimos de investimentos para os hospitais brasileiros entre os anos de 2006 e 2011. Para tanto, foram propostos os seguintes objetivos específicos: (a) desenvolver modelos DEA que expliquem a eficiência dos hospitais brasileiros; (b) identificar a influência relativa de fatores diversos sobre a eficiência obtida pelos hospitais; e (c) identificar os hospitais que apresentaram o maior nível de eficiência.

Este trabalho está dividido em cinco seções (contando com esta Introdução). Na seção 2, apresenta-se uma revisão da literatura sobre conceitos essenciais para a adequada compreensão deste trabalho. Em seguida, na seção 3, descreve-se a metodologia empregada no desenvolvimento do estudo. Na seção 4, descrevem-se e analisam-se os resultados do estudo. Por fim, a 
seção 5 apresenta as conclusões do artigo.

\section{REVISÃO DA LITERATURA}

\subsection{Análise financeira}

Silva (2012) afirma que a utilização de indicadores financeiros possibilita, por exemplo: (i) a análise, em um período específico de simultâneas organizações de um mesmo segmento, região geográfica e porte para realizar comparações entre elas ou verificar se as mesmas estão de acordo com as normas de seu segmento; e (ii) a análise de evolução histórica de uma organização, realizando comparações anuais. Outra importante utilidade dos indicadores financeiros é a previsão de crise da organização, baseado nos indicadores de liquidez, lucratividade e solvência da empresa (LIN et al., 2011). Ressalva-se que, conforme Gitman (2010), um índice isolado não é suficiente para se determinar o desempenho de uma organização. Contudo, o mesmo autor também afirma que, para a análise de aspectos específicos, poucos indicadores são suficientes, fazendo que com seja preciso estabelecer critério para a escolha dos mais adequados entre todas as opções disponíveis.

Dentre os diversos indicadores financeiros existentes, os mais utilizados são os de liquidez, endividamento e rentabilidade (BIROLO, 2010). Souza et al. (2010) classifica os indicadores mais abordados na literatura como indicadores de: (i) Eficiência de ativos; (ii) Rentabilidade; (iii) Estrutura de Capital; (iv) Liquidez; e (v) Lucratividade.

A análise financeira, por mais que tenha resultados relevantes, não abrange todos os fatores que impactam na saúde financeira das organizações, pois elas podem criar resistência para fornecer informações complementares, além da hipótese de ocorrência de defasagem das informações das demonstrações contábeis (SILVA, 2012). Nesse sentido, Lin et al. (2011) destaca que a utilização dos indicadores não leva em consideração características não financeiras potencialmente influentes como, por exemplo, estilo de gestão da organização.

\subsection{Gestão financeira de hospitais}

Os hospitais podem ser vistos como organizações produtoras de serviços, os quais são passíveis de avaliações (MANZO et al., 2011). Salienta-se que a avaliação dessas organizações pode ser baseada em indicadores econômicos financeiros, tal como em empresas, por exemplo (ZELLER et al., 1996; GRUEN; HOWARTH, 2005).

Um ponto a ser considerado em hospitais é a sua gestão, que de acordo com Vendemiatti et al. (2007), ocorre baseada na relação entre as subculturas de especialistas técnicos (médicos, fisioterapeutas, enfermeiros etc.) e do pessoal administrativo. Conforme os autores supracitados, a subcultura pode ser entendida como "compreensão coletiva" de um subsetor dos integrantes da organização, que mantém interações constantes entre si e se identificam como um grupo distinto dentro de uma organização. Tais subgrupos, assim, empregam sua compreensão própria para formular diretrizes do que seria melhor para a equipe. Devido à hierarquia dos funcionários, tanto no hospital quanto na subcultura, funcionários podem tomar decisões por conta própria, o que pode atrapalhar as rotinas globais dos hospitais, dificultando mais ainda a gestão dessas organizações.

Por causa de sua complexidade, os hospitais possuem diversos problemas de gestão, um dos quais observados por Almeida (2009) foi que a administração dos hospitais estudados baseia-se em administração familiar, ou seja, para seleção dos gestores leva-se em consideração os vínculos pessoais e familiares e não a experiência da pessoa. Canazaro (2007) destaca o fato de 
que cargos importantes nessas organizações são ocupados por médicos, além de eles realizarem suas atividades rotineiras e liderarem grande parte dos processos organizacionais. Estes pontos levantados pelos autores mostram que os gestores dos hospitais preocupam-se com a resolução de problemas de curto prazo e maximização da utilização de recursos, deixando de fazer uso de ferramentas gerenciais para o auxílio da tomada de decisão no médio e longo prazos, tal como os indicadores financeiros. Diversos indicadores financeiros em hospitais são utilizados nos estudos nacionais e internacionais, mas sem uma conclusão de quais são os melhores indicadores que devem ser utilizados em pesquisas empíricas (CANAZARO, 2007). Em sua pesquisa, Souza et al. (2009) lista alguns mais adequados para a análise de organizações hospitalares, levando em consideração a disponibilidade das informações e a relevância do significado dos indicadores.

Bittar (2001) salienta a importância de se empregar indicadores financeiros para analisar hospitais, dentre os quais se destaca o retorno sobre o ativo (ROA). Este se refere ao resultado (lucro ou prejuízo) dividido sobre o ativo total da organização (MATARAZZO, 2010). Muitos autores também citam a importância de algumas medidas de desempenho tal como o lucro antes dos juros, tributos, depreciação e amortização (earnings before interests and taxes- EBIT). Silva (2012) conceitua o EBIT como uma medida de performance operacional, que considera as receitas operacionais líquidas, após a dedução dos custos e das despesas operacionais. Nesse sentido, a margem EBIT é um indicador de lucratividade obtido pela divisão do EBIT pela receita da organização.

No estudo destacado neste artigo foram empregadas algumas outras informações financeiras: ativo imobilizado, receitas e despesas operacionais. Segundo o Comitê de Pronunciamentos Contábeis (CPC), o ativo imobilizado é conceituado como um ativo tangível mantido para uso na produção ou fornecimento de mercadorias e serviços e que se espera utilizar por mais de um exercício social (CPC, 2009). Já a receita é conceituada como "aumentos dos benefícios econômicos durante o exercício social sob a forma de entrada de recursos ou aumento de ativos ou diminuição de passivos que resultam em aumentos do patrimônio líquido e que não sejam provenientes de aporte dos proprietários da entidade" (CPC, 2011). Por fim, as despesas operacionais podem ser compreendidas como gastos periódicos necessários para manter o funcionamento das operações da organização. ludícibus et al. (2010) cita como exemplos de despesas operacionais: salários dos funcionários, aluguéis de espaços e depreciação de equipamentos etc.

\subsection{Análise envoltória de dados (Data envelopment analysis - DEA)}

A DEA pode ser entendida como uma técnica de programação linear não paramétrica que avalia a eficiência de unidades tomadoras de decisão (Decision Making Units - DMUs) (YANG et al., 2012). Segundo Azevedo et al. (2012), uma DMU é uma unidade capaz de transformar inputs em outputs.

Segundo Valdmanis et al. (2008), o modelo DEA cria uma fronteira de eficiência por meio da resolução de problemas de programação linear que identificam as melhores DMUs no que se refere à maximização de seus resultados (outputs), dado seus recursos (inputs). Este modelo foi desenvolvido inicialmente por Charnes et al. (1978) e estendido por Banker et al. (1984) (CHUANG et al., 2011).

O modelo desenvolvido por Charnes et al. (1978) é conhecido como CRR e considera retornos constantes de escala, ou seja, pressupõe que um aumento nos inputs teria um efeito proporcional nos outputs. Por sua vez, o modelo de Banker et al. (1984), conhecido como BCC, considera retornos variáveis de escala, ou seja, não segue a pressuposição supracitada do modelo anterior.

Um detalhe importante do modelo diz respeito à orientação a inputs ou a outputs. De 
acordo com Mark et al. (2009), os modelos orientados aos inputs consideram que as grandezas de entrada (inputs) podem ser reduzidas sem se alterar a quantidade produzida (outputs), enquanto que os modelos orientados aos outputs consideram que a quantidade produzida pode ser maximizada sem se alterar a quantidade de insumos utilizada.

Conforme Ozcan et al. (2010), alterações necessárias para que as unidades ineficientes atinjam a fronteira de eficiência podem ser direcionadas à redução de recursos ou ao aumento de produtos. Além disso, modelos orientados aos inputs assumem que os gestores têm controle sobre os insumos, enquanto a orientação aos outputs assume que estes têm controle sobre os produtos.

O modelo DEA se baseia no conceito de eficiência relativa, que pode ser definida como a razão entre a soma ponderada dos outputs e a soma ponderada dos inputs (COOPER et al., 2004 apud CHIU; LU; KAO, 2011). Segundo Yang et al. (2012), essa modelagem converte os inputs e outputs em medidas de eficiência relativa (i.e., comparativa), além de construir uma fronteira determinística, classificando as DMUS em eficientes ou ineficientes. As DMUs que se encontram sobre a fronteira têm eficiência relativa igual a $1(100 \%)$, enquanto as DMUs localizadas abaixo da fronteira (i.e., ineficientes) apresentam medidas de eficiências que variam no intervalo $[0,1]$ (LOBO et al., 2011).

De acordo com Saurin et al $(2013$, p. 27) o modelo DEA foi aplicado "inicialmente em instituições públicas em que a dificuldade de alocação de preços a insumos e produtos impossibilitava a mensuração de sua eficiência". Atualmente o modelo DEA tem uma ampla gama de aplicabilidades nas mais diversas áreas (CHIU et al., 2011), tais como: transportes (AZEVEDO et al., 2012), clubes de futebol (BARROS et al., 2010; DANTAS; BOENTE, 2011) e hospitais (CHILINGERIAN; SHERMAN, 2010). Dentre os trabalhos relacionados a este último tema e foco deste trabalho podem ser citados os estudos nacionais de Lobo et al. (2011) e Guerra et al. (2012), e, como internacionais, os trabalhos de El-Jardali et al. (2011), Sulku (2011) e Yang et al. (2012).

\section{METODOLOGIA}

A pesquisa pode ser classificada como de natureza descritiva com um enfoque quantitativo. Os dados empregados na pesquisa foram secundários. Os dados operacionais dos hospitais foram coletados com base no Banco de Dados do Sistema Único de Saúde (DATASUS) e, os financeiros, por meio das demonstrações contábeis dos hospitais disponíveis na imprensa oficial do estado onde a organização tem sua sede, ou mesmo nos sites das referidas organizações. Ao todo, informações de 31 hospitais foram reunidas e analisadas em um período de seis anos, de 2006 até 2011 Trata-se, assim, de uma amostra não probabilística por conveniência (ALENCAR, 2007). As organizações selecionadas apresentam características bem diversas no que tange à natureza jurídica, porte, localização geográfica e número de leitos. Salienta-se que os dados contidos nas demonstrações contábeis dos hospitais foram atualizados segundo o Índice Nacional de Preços ao Consumidor Amplo (IPCA) mensurado pelo Instituto Brasileiro de Geografia e Estatística (IBGE) para o mês de dezembro de 2012 (IBGE, 2013).

A seguir, apresentam-se os hospitais da amostra assim como o seu código de análise: Albert Einstein (DMU 1), Associação de Beneficência e Filantropia São Cristóvão (DMU 2), Fundação Ary Flauzino para Pesquisa e Controle Câncer - Fundação do Câncer - RJ (DMU 3), Fundação de Ensino e Pesquisa de Uberaba (DMU 4), Fundação Zerbini (DMU 5), Hospital 9 de Julho (DMU 6), Hospital A.C. Camargo (DMU 7), Hospital das Clínicas da Faculdade de Medicina de Ribeirão 
Preto USP (DMU 8), Hospital e Maternidade Nossa Senhora de Lourdes (DMU 9), Hospital Erastor Gaertner (DMU 10), Hospital Geral de Guarulhos (DMU 11), Hospital Geral de Pedreira (DMU 12), Hospital Metropolitano de Urgência e Emergência (DMU 13), Hospital Novo Atibaia (DMU 14), Hospital Regional de Franca S.A. (DMU 15), Hospital Samaritano (DMU 16), Hospital Santa Rita (DMU 17), Hospital São José (DMU 18), Hospital Vera Cruz (DMU 19), Real e Benemérita Associação Portuguesa de Beneficência (DMU 20), Santa Casa de Belo Horizonte (DMU 21), Santa Casa de Maceió (DMU 22), Santa Casa de Misericórdia de Fortaleza (DMU 23), Santa Casa de Misericórdia de Porto Alegre (DMU 24), Santa Casa de Misericórdia de São Francisco (DMU 25), Santa Casa de Misericórdia de Tatuí (DMU 26), Santa Casa de Misericórdia de Valparaíso (DMU 27), São Domingos (DMU 28), Sociedade Assistencial Bandeirantes (DMU 29), Sociedade Brasileira e Japonesa de Beneficência Santa Cruz (DMU 30), SPDM - Associação Paulista para o Desenvolvimento da Medicina Hospital São Paulo (DMU 31).

Para a análise dos dados foram empregadas diferentes técnicas: estatística descritiva, teste de Kruskal-Wallis e o modelo DEA. Conforme Carlos (2004), a estatística descritiva consiste na elaboração de tabelas, gráficos e medidas que são exploradas no intuito de facilitar o entendimento das informações. Por sua vez, de acordo com Maroco (2010), o teste de Kruskal-Wallis é definido como uma alternativa não paramétrica para testar se duas ou mais amostras proveem de populações semelhantes ou de populações diferentes. Por fim, empregou-se a análise DEA para se obter os escores de eficiência comparativa entre os hospitais e assim identificar os fatores que determinam os padrões ótimos de investimento. No estudo descrito neste trabalho optou-se por utiliza o modelo BBC do DEA orientado a outputs. O modelo BBC considera retornos variáveis de escala, ou seja, ele desconsidera a relação de proporcionalidade entre inputs e outputs, admitindo que as DMUs analisadas tenham portes diferentes. Por sua vez, a orientação a outputs considera que a quantidade produzida pode ser maximizada sem se alterar a quantidade de insumos utilizada. Para a operacionalização da técnica, empregou-se o Sistema Integrado de Apoio à Decisão (ÂNGULO MEZA et al., 2005).

\section{RESULTADOS}

\section{1. desenvolvimento dos modelos dea}

Foram propostos dois modelos DEA na pesquisa: Modelo I (Quadro 1) e Modelo II (Quadro 2). Os inputs e os outputs de cada modelo foram propostos para identificar padrões ótimos de investimentos nos hospitais. Em ambos os modelos, os inputs foram o "Investimento em ativo imobilizado" e as "Despesas operacionais". Tal como apresentado anteriormente, o primeiro representa o investimento dos hospitais (a longo prazo) em estrutura para garantir suas operações, o segundo, por sua vez, classifica-se como gastos periódicos necessários para manter as operações cotidianas.

Já os outputs do Modelo I foram a "Receita" e o "Número de leitos". Assim, este modelo destacará como eficientes os hospitais que conseguirem aumentar o número de leitos e a sua receita com o menor investimento em ativo imobilizado e menores gastos periódicos. Ademais, os outputs do Modelo II foram a "Margem EBIT" e o "ROA". Neste modelo visou-se avaliar a eficiência dos hospitais em termos de lucratividade e rentabilidade, ou seja, serão considerados eficientes os hospitais que apresentarem os melhores resultados em relação aos investimentos operacionais e gastos periódicos. 
Quadro 1: Inputs e Outputs do Modelo I.

\begin{tabular}{|c|c|}
\hline Inputs & Outputs \\
\hline $\begin{array}{c}\text { Investimento em ativo imobilizado } \\
\text { Despesas operacionais }\end{array}$ & Receita \\
& Número de leitos \\
\hline
\end{tabular}

Fonte: Elaborado pelos autores

Quadro 2: Inputs e Outputs do Modelo II

\begin{tabular}{|c|c|}
\hline Inputs & Outputs \\
\hline Investimento em ativo imobilizado & ROA \\
Despesas operacionais & Margem EBIT \\
\hline
\end{tabular}

Fonte: Elaborado pelos autores

Antes do prosseguimento com os modelos foram levantadas as características gerais para as variáveis conforme a Tabela 1. Os valores estão expressos em termos de média dos seis anos estudados, quais sejam de 2006 a 2011, considerando todos os hospitais da amostra.

Por meio da Tabela 1 percebe-se que os dados da amostra são heterogêneos, pois o Coeficiente de Variação foi superior à unidade com exceção da Receita. Também se nota que em média o ROA e a Margem EBIT apresentaram valores negativos evidenciando problemas financeiros para os hospitais estudados. Ressalva-se que o Coeficiente de Variação não foi calculado para o ROA e a Margem EBIT dado que estas variáveis apresentaram média negativa o que prejudica a análise desse indicador de dispersão.

Tabela 1: Aspectos gerais das variáveis

\begin{tabular}{|c|c|c|c|c|c|c|}
\hline \multirow[t]{2}{*}{ Estatística } & \multicolumn{2}{|c|}{ Inputs Modelos I e II } & \multicolumn{2}{|c|}{ Outputs Modelo I } & \multicolumn{2}{|c|}{ Outputs Modelo II } \\
\hline & $\begin{array}{l}\text { Investimento } \\
\text { em Ativo Imo- } \\
\text { bilizado }\end{array}$ & $\begin{array}{c}\text { Despesas } \\
\text { operacionais }\end{array}$ & Receita & $\begin{array}{l}\text { Número } \\
\text { de leitos }\end{array}$ & ROA & $\begin{array}{l}\text { Margem } \\
\text { EBIT }\end{array}$ \\
\hline Média & $64.922 .473,86$ & $17.437 .563,99$ & $139.524 .236,43$ & 253,748 & $-9,52 \%$ & $-2,21 \%$ \\
\hline Desvio Padrão & $82.602 .951,27$ & $20.729 .397,84$ & $135.406 .582,65$ & 255,76 & 0,2082 & 0,2769 \\
\hline $\begin{array}{l}\text { Coeficiente } \\
\text { de Variação }\end{array}$ & 1,2723 & 1,1888 & 0,9705 & 1,0079 & & \\
\hline
\end{tabular}

Fonte: dados da pesquisa

\section{2. apresentação e discussão do modelo I}

No Modelo I, a média de eficiência geral foi de 0,71 no período. As unidades com melhores escores em todos os anos foram as DMUs 4, 11, 16 e 20. De acordo com a Figura 1, observa-se que a maior média de eficiência ocorreu em $2011(0,76)$ e a pior média ocorreu no ano de $2006(0,67)$. Ainda assim como o DEA estabelece essa medida de forma comparativa entre as DMUs é preciso verificar ano a ano os fatores determinantes para esses resultados. 
Figura 1: Eficiência média - Modelo I.

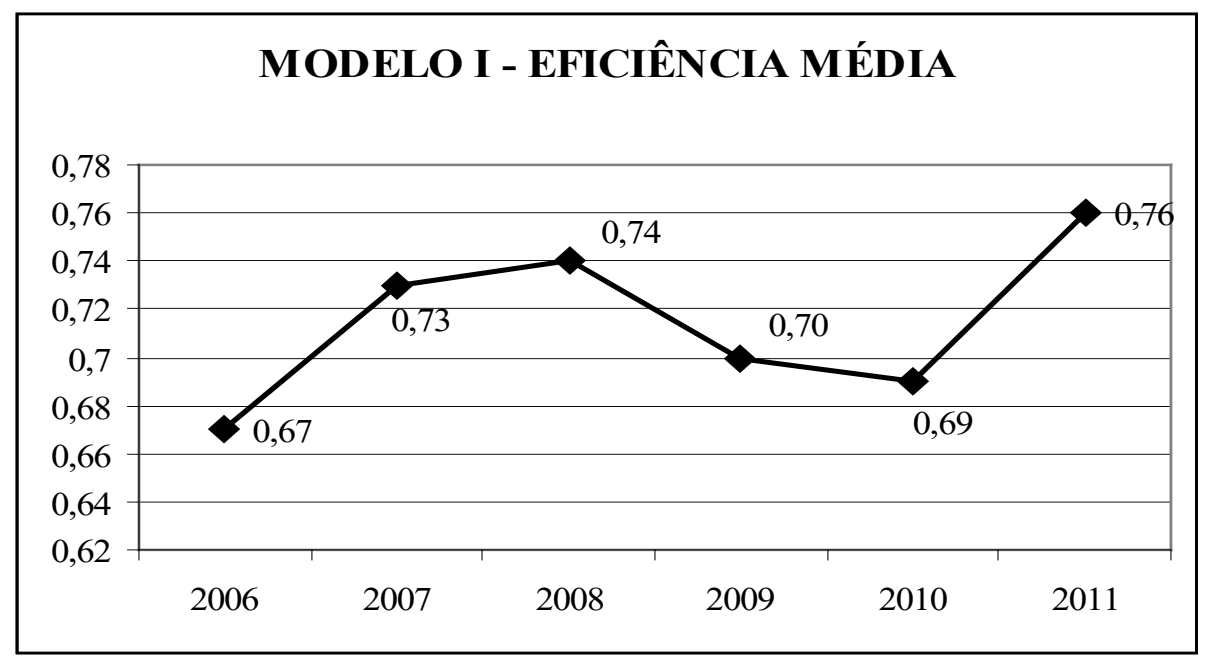

Fonte: Elaborado pelos autores

Para o ano de 2006, foi verificado que as DMUs 4, 7, 11, 16, 18, 20, 24, 27 e 29 foram apontadas como eficientes pelo modelo e assim apresentaram o melhor escore de eficiência $(1,00)$. Por sua vez, as DMUs 25,26 e 30 evidenciaram os menores valores, pois demonstraram um montante de receita baixo (entre os cinco menores da amostra) considerando também os inputs do Modelo I.

No ano de 2007, os hospitais eficientes foram as DMUs 4, 6, 8, 11, 16, 18, 20, 23, 24, 27, 29 e 31, praticamente os mesmos do ano de 2006. Isto se deve ao fato que as variáveis consideradas no modelo sofrem relativamente pouca alteração em um ano, especialmente no que diz respeito ao Investimento em Imobilizado e o Número de Leitos. Por outro lado, as DMUs 15, 25 e 28 obtiveram os menores valores de eficiência dado principalmente ao número de leitos reduzido como também ao baixo desempenho em relação as receitas.

Os anos de 2008 e 2009 apresentaram comportamentos semelhantes para a eficiência dos hospitais. As mesmas DMUs foram apontadas como eficientes tanto em 2008 como em 2009: 4, 8, 11, 16, 18, 20, 21, 23, 27 e 31. Por sua vez, as DMUs que apresentaram menores valores em 2008 foram as 14, 25 e 28 e em 2009 as DMUs 25, 27 e 28.

No ano de 2010 as DMUs 4, 8, 11, 13, 16, 20, 21, 23, 29 foram eficientes, e os menores valores de eficiência foram evidenciados nas DMUs 25, 27 e 28. Por fim, no ano de 2011 as DMUs 4, 8, 11, 20, 21, 23, 29, 30 e 31 apresentaram o máximo de eficiência (1,00). E as DMUs 14, 25 e 28 mostraram valores mínimos de eficiência.

Destaca-se que analisando o comportamento das DMUs eficientes em todos os anos da amostra, ou seja, as DMUs 4, 11, e 20 verificou-se que as mesmas apresentaram comportamento diferente. A DMU 20 apresentou altos valores de investimento em imobilizado e de despesas. Porém, foi a unidade com a maior receita em todos os anos estudados, assim como um dos maiores valores para número de leitos. Dessa forma, sua eficiência foi devido principalmente aos produtos apresentados. Por outro lado, as DMUs 4 e 11 não apresentaram valores tão altos para a receita, mas mantiveram bons valores para número de leitos e despesas e investimento em imobilizado baixos assim essas são organizações que se destacaram pelo bom aproveitamento dos recursos. 
Em relação aos piores hospitais, destaca-se os hospitais 25 e 28 que obtiveram resultados ruins em todos os anos estudados. No hospital 25 isso ocorreu principalmente pelo número baixo de leitos combinado com o desempenho insatisfatório em relação à receita. Além disso, não apresentou um alto valor para despesas e investimento em ativo imobilizado, ou seja, o principal motivo para o baixo resultado da eficiência foram os produtos e não os insumos. No caso do hospital 28 o mesmo também apresentou baixo resultado em relação aos produtos, mas ao mesmo tempo continha valores expressivos para as despesas e principalmente em relação ao investimento em imobilizado. Assim, esse hospital não é eficiente na aplicação dos inputs para a produção dos outputs, pois os demais hospitais apresentam uma melhor relação entre estes fatores.

É importante ressaltar que se verificou diferença estatística significativa (a menos de 1\%) considerando a natureza jurídica dessas organizações (i.e., públicos, privados com fins lucrativos e filantrópicos) e a eficiência obtida pelo Modelo I conforme a Tabela 2. Ademais, também se observou diferença estatística significativa (a menos de 1\%) entre a eficiência dos hospitais e o seu estado de origem.

Tabela 2 - Resultado do teste de Kruskal-Wallis Modelo I.

\begin{tabular}{ccc}
\hline Variável & Natureza Jurídica & Estado \\
\hline Estatística do Qui-quadrado & 21,903 & 34,886 \\
\hline P-valor & 0,000 & 0,000
\end{tabular}

Fonte: dados da pesquisa

Os hospitais privados com fins lucrativos obtiveram um resultado inferior em relação aos demais (tal como apresentado na Figura 2). Os hospitais dos estados do Ceará e Minas Gerais obtiveram resultados de eficiência superiores aos do estado do Paraná (vide Figura 3).

Figura 2: Eficiência média segundo a natureza jurídica- Modelo I.

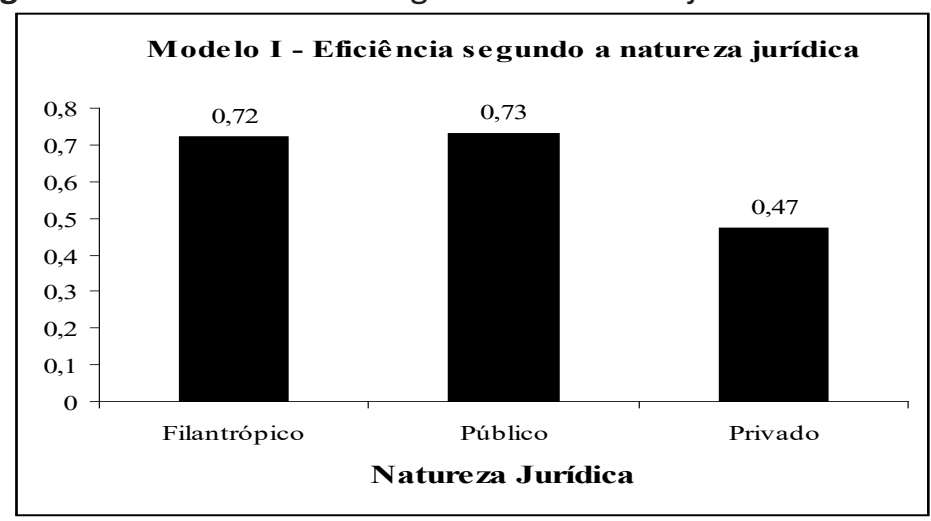

Fonte: Elaborado pelos autores 
Figura 3: Eficiência média segundo a unidade da federação - Modelo I.

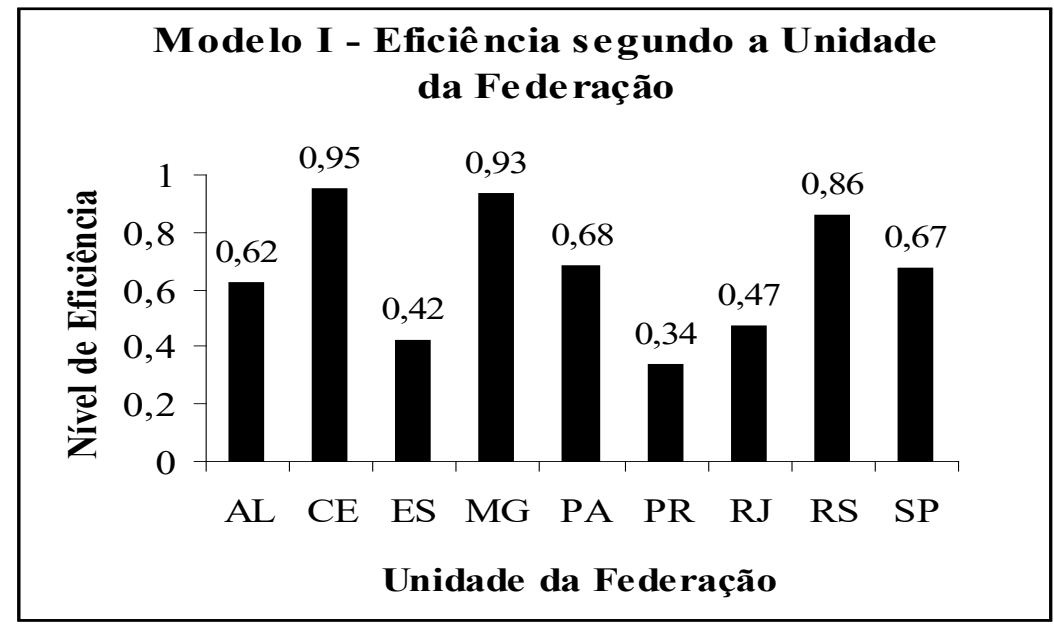

Fonte: Elaborado pelos autores

\section{3. apresentação e discussão do modelo II}

No Modelo II a média apresentou um comportamento diferente do que no Modelo I, com um valor máximo para 2009, a média geral de eficiência foi de 0,87 . Neste segundo modelo, as DMUs 16 e 27 indicaram melhores resultados, ou seja, hospitais diferentes em relação ao Modelo I onde as melhores DMUs foram a 4, 11, e 20, dado a diferença na ênfase do Modelo II que é voltado a determinação da eficiência considerando índices de lucratividade.

Figura 5: Eficiência média - Modelo II.

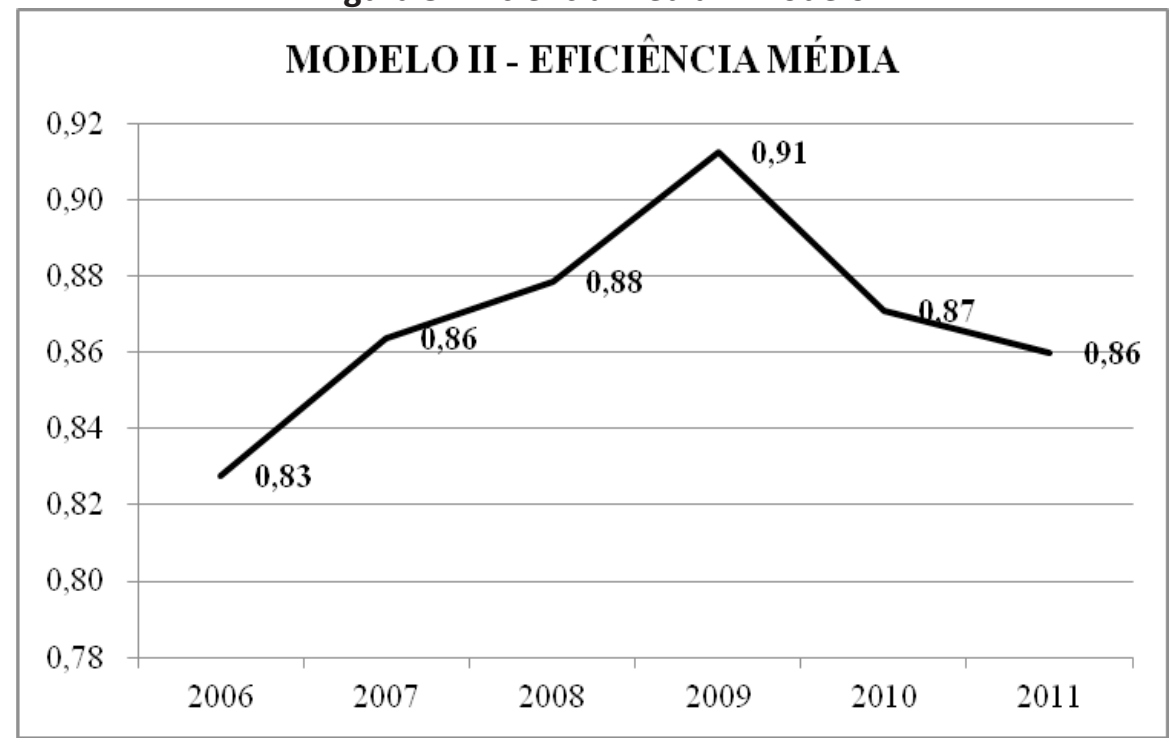

Fonte: Elaborado pelos autores 
Para o ano de 2006, as DMUs 4, 9, 16, 18 e 27 apresentaram o máximo de eficiência $(1,0)$, enquanto os menores valores foram mostrados nas DMUs 2, 5 e 25. Em 2007, as DMUs 4, 7, $8,9,16,17,18,27$ e 30 obtiveram os resultados máximos, já as DMUs 21, 25 e 31 apresentaram os menores valores de eficiência do ano citado.

Percebe-se que algumas DMUs, como a 4 e a 18, se destacaram em ambos os modelos, ainda assim a DMU 9 não havia tido destaque no primeiro modelo, mas foi apontada como eficiente pelo Modelo II. Isso ocorreu, pois no primeiro modelo este hospital apresentava valores médios para as vaiáveis inputs e outputs, porém no Modelo II verificou-se que o mesmo obteve valores comparativamente altos para Margem EBIT.

Em 2008 as DMUs 4, 7, 8, 9, 15, 16, 18 e 27 exibiram o máximo de eficiência anual $(1,00)$, já as DMUs 3, 24 e 26 evidenciaram os menores valores. Em 2009, as DMUs 7, 8, 11, 16, 18, 27 e 30 apresentaram o máximo de eficiência e, por sua vez, os mais baixos valores foram os destacados pelas DMUs 5, 23 e 25.

Dessa forma, verifica-se que as piores DMUs em 2008 foram diferentes das piores DMUs em 2009. Essa discrepância foi motivada pela alteração nas variáveis despesas operacionais, Margem EBIT e ROA, ou seja, as DMUs 5, 23 e 25 que pioraram seus índices em relação a essas variáveis entre 2008 e 2009. Nota-se que no geral as diferenças nas eficiências com intervalo de um ano são maiores no Modelo II, pois o mesmo usa variáveis que se alteram com mais facilidade (Margem EBIT e ROA) comparadas com o Modelo I (Número de Leitos e Receita).

No ano de 2010, as DMUs eficientes foram: 2, 4, 7, 9, 11, 13, 16, 17, 18, 27 e 28, por outro lado, as DMUs 23, 25 e 31 apresentaram os valores mais baixos de eficiência. Por fim, em 2011, as DMUs 7, 8, 11, 17, 28 e 30 revelaram o máximo de eficiência, já as DMUs 12, 21 e 31 obtiveram os piores valores.

O elevado número de DMUs eficientes para o ano de 2010 se deve ao fato de que nenhuma organização se destacou muito além das demais. Assim, por exemplo, o hospital 4 que obteve menor valor para as Despesas Operacionais, mas também apresentou baixo valor para Margem EBIT, como também o hospital 7 detinha o maior valor para o ROA mas também apontou um alto valor para as Despesas Operacionais. É preciso considerar que o Modelo DEA estabelece a eficiência de forma comparativa entre as DMUs da amostra, assim aquelas que apresentarem os melhores resultados serão eficientes, mas essa medida é limitada para as DMUs da composição da amostra.

Considerando o comportamento das DMUs 16 e 27 que obtiveram os melhores resultados de eficiência para o Modelo II constatou-se que ambas apresentaram valores comparativamente altos para a Margem EBIT em todos os anos estudados. Contudo a DMU 16 também se destacou por revelar um baixo valor para as Despesas Operacionais em todo o período e por sua vez a DMU 27 também se fez notar por indicar um baixo valor para investimento em imobilizado em todo o período.

Salienta-se que não foi verificada diferença estatística significativa (a menos de 1\%) considerando a natureza jurídica dessas organizações (i.e., públicos, privados com fins lucrativos e filantrópicos) e a eficiência obtida no Modelo II, conforme a Tabela 3. Por outro lado, constatou-se diferenças significantes estatisticamente (a menos de $1 \%$ ) ao se considerar o estado de origem do hospital e a eficiência. 
Tabela 3 - Resultado do teste de Kruskal-Wallis Modelo II.

\begin{tabular}{ccc}
\hline Variável & Natureza Jurídica & Estado \\
\hline Estatística do Qui-quadrado & 3,808 & 23,793 \\
\hline P-valor & 0,149 & 0,002 \\
\hline Fonte: dados da pesquisa & &
\end{tabular}

Conforme a figura 6, os hospitais privados apresentaram uma média de eficiência maior se comparado aos demais. Tendo em vista a natureza jurídica destes, uma possível explicação é que tais organizações aplicam práticas de gestão com um enfoque maior no lucro, ao passo que as demais têm por intuito principal a execução dos procedimentos médicos para o tratamento dos pacientes.

Figura 6: Eficiência média segundo a natureza jurídica- Modelo II.

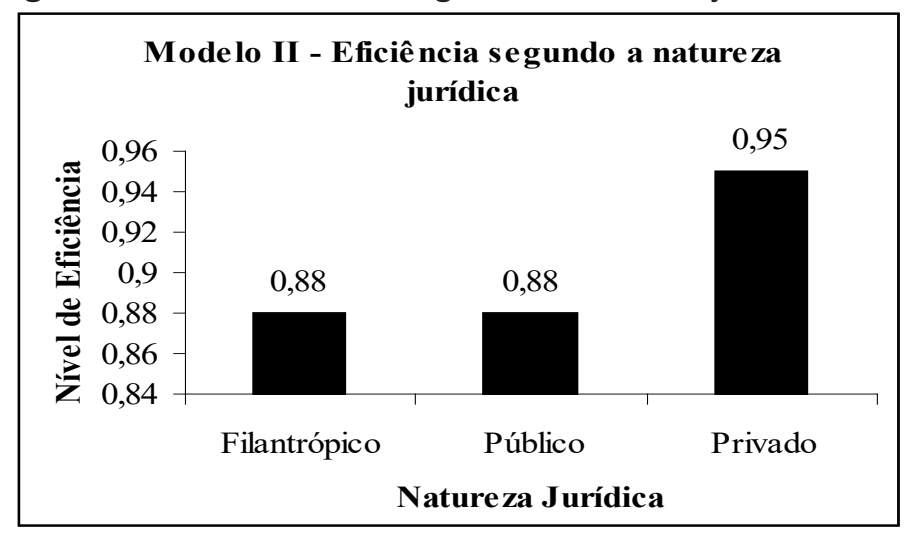

Fonte: Elaborado pelos autores

Os hospitais do Espírito Santo apresentaram resultados de eficiência superiores aos do estado do Ceará, tal como exposto na Figura 7. Neste caso, os valores não são conclusivos, uma vez que a amostra não é probabilística, mas, de qualquer forma, espera-se que fatores geográficos impactem a eficiência hospitalar.

Figura 7: Eficiência média segundo a unidade da federação - Modelo II.

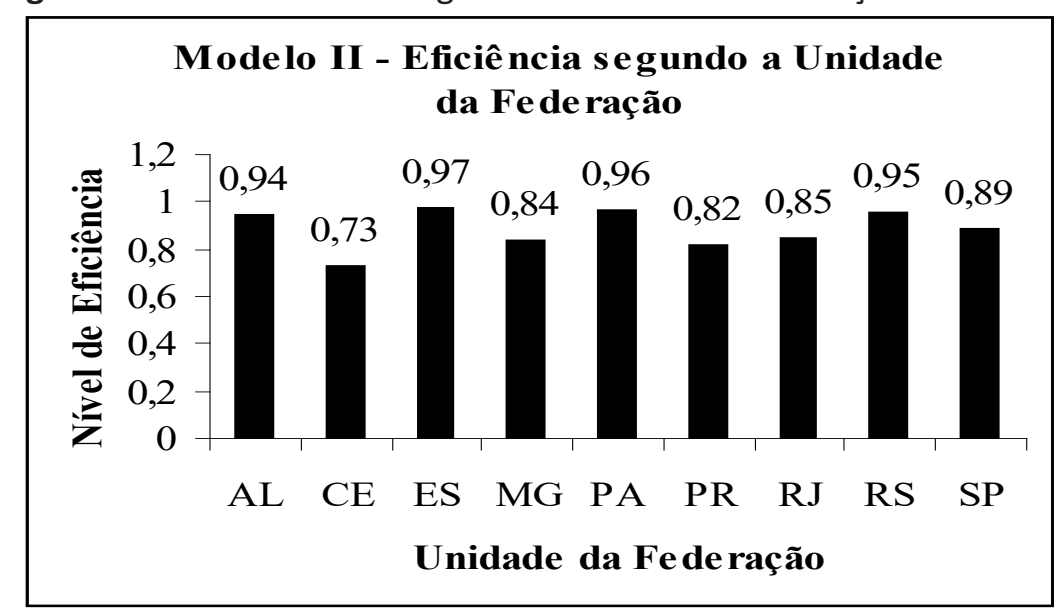

Fonte: Elaborado pelos autores 


\section{CONCLUSÕES}

Este artigo apresentou os resultados de uma pesquisa que objetivou identificar padrões ótimos de investimentos para os hospitais brasileiros entre os anos de 2006 e 2011. Nesse sentido, foram desenvolvidos e analisados dois modelos baseados na análise DEA.

Constatou-se que o porte, a natureza jurídica e a unidade da federação de origem influenciaram a eficiência dos hospitais entre os anos de 2006 e 2011. No Modelo I, que analisou a eficiência em termos de receita e número de leitos, as organizações públicas e filantrópicas apresentaram um resultado superior aquelas com fins lucrativos. Por outro lado, estas últimas organizações apresentaram resultados melhores ao se mensurar a sua eficiência em termos de lucratividade e rentabilidade (Modelo II). Tal resultado pode ser devido ao fato de que os hospitais sem fins lucrativos têm por missão o atendimento a um maior volume de pessoas em suas unidades, enquanto as organizações com fins lucrativos empregam os seus recursos com forte orientação a resultados financeiros.

Por meio da análise da eficiência dos hospitais percebeu-se que alguns hospitais se destacam por apresentarem um alto valor para os outputs considerando os outros, porém obtiveram melhores resultados dado principalmente ao baixo valor relativo dos inputs. O mesmo fenômeno se verificou para as organizações com piores resultados. Em alguns casos também, muitos hospitais foram apontados como eficientes pelo fato de formarem um grupo homogêneo que se destacou dos demais. É importante salientar este ponto, pois o modelo DEA estabelece a eficiência de forma comparativa e assim a(s) melhor(es) DMU(s) é(são) eficiente(s) independente se os resultados são os desejáveis. Assim, na estatística descritiva constatou-se que as médias do ROA e da Margem EBIT são negativas, dado que muitos hospitais apresentaram prejuízo no período.

Verificou-se que cinco hospitais apresentaram padrões de investimentos que podem ser considerados ótimos segundo os modelos desenvolvidos. No caso do Modelo I, os hospitais foram os seguintes: Fundação de Ensino e Pesquisa de Uberaba, Hospital Geral de Guarulhos, Hospital Samaritano e Real e Benemérita Associação Portuguesa de Beneficência. Todos se localizam em estados da região sudeste do Brasil, possuem mais de 100 leitos e são filantrópicos (com exceção do Hospital Geral de Guarulhos, que é público). Por sua vez, no Modelo II, os hospitais que apresentaram padrões ótimos de desempenho foram os seguintes: Hospital Samaritano e Santa Casa de Misericórdia de Valparaíso. Ambos são filantrópicos e se encontram no Estado de São Paulo. Todavia, ao contrário do modelo anterior, a Santa Casa de Misericórdia de Valparaíso apresentou apenas 55 leitos ao longo de todo o período de análise.

Devido à relevância essencial dos hospitais para o sistema de saúde, é importante que haja uma constante busca pela melhoria na gestão dos serviços de saúde, como evidenciam autores como Ozgulbas e Koyuncugil (2009) e Guerra (2011). Dessa forma, a análise descrita neste artigo contribui significativamente para a pesquisa da eficiência dos hospitais, especialmente no que tange a investimentos. $O$ emprego do modelo DEA pode auxiliar significativamente no estudo dessas organizações essenciais, tal como ratifica Guerra et al. (2012). Ademais, os modelos propostos apresentaram resultados coerentes, significativos e aplicáveis em outros contextos.

Como limitações da pesquisa, podem-se citar o fato de que o Modelo DEA não permite generalizados para além da amostra, assim os hospitais apontados como eficientes nos Modelos I e II não necessariamente seriam em comparação a outros, como também na utilização de outras variáveis. Estudos futuros poderiam aumentar a amostra empregada, assim como enfocar uma única região do país. Além disso, tais pesquisas poderiam aplicar os modelos desenvolvidos de 
forma a corroborar ou não os resultados obtidos.

\section{REFERÊNCIAS BIBLIOGRÁFICAS}

ALENCAR, E. Pesquisa em turismo. Lavras: FAEPE, 2007.

ALMEIDA, M. A. V. Modelo da gestão financeira em hospitais privados de pequeno e médio porte da cidade do Rio de Janeiro: uma análise focada na visão dos gestores. 2009, 78 f. Dissertação (Mestrado em Gestão de Empresas) - Instituto universitário de Lisboa - ISTCE. Lisboa, 2009.

ANCARANI, A. A.; MAURO, C. D. \& GIAMMANCO, M. D. The impact of managerial and organizational aspects on hospital wards' efficiency: Evidence from a case study. European Journal of Operational Research, v. 194, p. 280-293, 2009.

ÂNGULO MEZA, L. et al. ISYDS - Integrated System for Decision Support (SIAD - Sistema Integrado de Apoio à Decisão): a software package for data envelopment analysis model. Pesquisa Operacional, Rio de Janeiro, v. 25, n. 3, 2005, p. 493-503.

AZEVEDO, G.H.I. et al. Uso de análise envoltória de dados para mensurar eficiência temporal de rodovias federais concessionadas. Journal of Transport Literature, v. 6, n. 1, 2012, p.37-56.

BANKER R. D.; CHARNES, R. F. \& COOPER, W. W. Some models for estimating technical and scale inefficiencies in data envelopment analysis. Management Science, v. 30, p. 1078-1092, 1984.

BARROS, C. P.; ASSAF, A.; SÁ-EARP, F. Brazilian football league technical efficiency: A Simar and Wilson approach. Journal of Sports Economics, v. 11, n. 6, p. 641-651, 2010. 
BIROLO, P. B. Análise de crédito por meio de modelos quantitativos para a previsão de insolvência: um estudo de caso em uma empresa do setor cerâmico. 2010. 67f. Trabalho de Conclusão do Curso de Ciências Contábeis- Universidade do Extremo Sul Catarinense -UNESC, Criciúma - SC, 2010.

BITTAR, O. J. N. V. Indicadores de qualidade e quantidade em saúde. Revista de Administração da Saúde, São Paulo, v. 3, n. 12, p. 21-28, 2001.

CANAZARO, M. P. Desempenho econômico-financeiro de nosocômios brasileiros: uma análise comparativa de hospitais com e sem fins lucrativos. 2007, 99 f. Dissertação (Mestrado em Administração) - Universidade do Vale do Itajaí - UNIVALI, Biguaçu, 2007.

CARLOS, F. A. Gestão de satisfação e fidelidade do cliente: um estudo com turistas em hotéis. 2004. 87 f. Dissertação (Mestrado em Engenharia de Produção)- Universidade Federal do Rio Grande do Norte, Natal, 2004.

CHARNES, A.; COOPER, W. W. \& RHODES, E. Measuring the efficiency of decision making units. European Journal of Operational Research, v. 2, n. 6, p. 429-444, 1978.

CHILINGERIAN, J. A. \& SHERMAN, H. D. Health-Care Applications: From Hospitals to Physicians, from Productive Efficiency to Quality Frontiers. In: COOPER, W. W.; SEIFORD, L. M. \& ZHU, J. (Org.) Handbook on data envelopment analysis. 2 ed. [S. L.]: Springer, 2010, p. 445-494.

CHIU, C.; LU, C. \& KAO, L. Efficiency measurement using independent component analysis and data envelopment analysis. European Journal of Operational Research, v. 210, p. 310-317, 2011.

CHUANG, C.; CHANG, P. \& LIN, R. An Efficiency Data Envelopment Analysis Model Reinforced 
by Classification and Regression Tree for Hospital Performance Evaluation. Journal of Medical Systems, v. 35, p. 1075-1083, 2011.

COMITÊ DE PRONUNCIAMENTOS CONTÁBEIS - CPC. Ativo imobilizado. 2009. Disponível em: http://www.cpc.org.br/pdf/CPC\%2027.pdf. Acesso em: 12 jun. 2011.

Pronunciamento Conceitual Básico (R1) Estrutura Conceitual para Elaboração e Divulgação de Relatório Contábil-Financeiro. 2011. Disponível em: http://www.cpc.org.br/pdf/ CPC00_R1.pdf. Acesso em: 12 jun. 2011.

DANTAS, M. G. S.; BOENTE, D. R. A eficiência financeira e esportiva dos maiores clubes de futebol europeus utilizando a análise envoltória de dados. Revista de Contabilidade e Organizações, v. 5 n. 13, p. 75-90, 2011.

EL-JARDALI, F. et al. Design, implementation and scaling up of the balanced scorecard for hospitals in Lebanon: Policy coherence and application lessons for low and middle income countries. Health Policy, v. 103, p. 305-314, 2011.

GITMAN, L. J. Princípios de Administração Financeira.12 ed. Pearson Education, 2010.

GRUEN, R. \& HOWARTH, A. Managing health services finance. 1a Ed. London: London School of Hygiene and Tropical Medicine, 2005.

GUERRA, M. Análise de desempenho de organizações hospitalares. 2011. 144 f. Dissertação (mestrado) - Universidade Federal de Minas gerais, Centro de Pós-Graduação e Pesquisas em Contabilidade e Controladoria, Belo Horizonte, 2011. 
GUERRA, M.; SOUZA, A. A. \& MOREIRA, D. R. Performance analysis: a study using data envelopment analysis in 26 Brazilian hospitals. Journal of Health Care Finance, v. 38, n. 4, p. 19-35, 2012.

INSTITUTO BRASILEIRO DE GEOGRAFIA E ESTATÍSTICA - IBGE. Índices de preços ao consumidor - IPCA e INPC. 2013. Disponível em: <http://www.ibge.gov.br/home/estatistica/indicadores/ precos/inpc_ipca/ipca-inpc_201207_1.shtm>. Acesso em: 12 jun. 2013

IUDíCIBUS, S. et al. Manual de Contabilidade Societária: aplicável a todas as sociedades : de acordo com as normas internacionais e do CPC. São Paulo, SP: Atlas, 2010.

LIN, F. et al. Financial ratio selection for business crisis prediction. Expert Systems with Applications, v. 38, p. 15094-15102, 2011.

LOBO, M. S. C. et al. Influência de fatores ambientais na eficiência de hospitais de ensino. Epidemiologia e Serviços de Saúde, Brasília, v. 20, n. 1, p. 37-45, 2011.

MANZO, B.F. et al. As percepções dos profissionais de saúde sobre o processo de acreditação hospitalar. Revista Enfermagem UERJ, Rio de Janeiro, v. 19, n. 4, p. 571-576, 2011.

MARK, B. A. et al. An Examination of Technical Efficiency, Quality, and Patient Safety in Acute Care Nursing Units. Policy, Politics, \& Nursing Practice, v. 10, n. 3, 2009, p. 180-186.

MAROCO, J. Análise estatística: com utilização do SPSS. 3. ed. Lisboa: Edições Sílabo, 2010. 
MATARAZZO, D. C. Análise financeira de balanços: abordagem básica. São Paulo: Atlas, 2010.

MEDEIROS, S. E. R. et al. Logística hospitalar: um estudo sobre as atividades do setor de almoxarifado em hospital púbico. Revista de Administração da UFSM, Santa Maria, v.2, n.1, p. 59-79, jan./abr. 2009.

NOVARETTI, M. C. Z.. aplicação da Análise Causa Raiz como ferramenta na gestão de segurança hospitalar. Revista de Administração da UFSM, Santa Maria, v. 7, número 3, p. 442-452, SET. 2014

O'DWYER, G. et al. The current scenario of emergency care policies in Brazil. BMC Health Services Research, 2013, p. 13-70.

OZCAN, Y. A. et al. Evaluating the performance of Brazilian university hospitals. Annals of Operations Research, n. 178, p. 247-261, 2010.

OZGULBAS, N. \& KOYUNCUGIL, A. S. Financial profiling of public hospitals: an application by data mining. International Journal of Health Planning and Management, v. 24, p. 69-83, 2009.

SAURIN, V. et al. Medidas de eficiência e retorno de Investimento: um estudo nas distribuidoras de energia elétrica brasileiras com base em Data Envelopment Analysis, Índice de Malmquist e ROI. Rev. Adm. UFSM, Santa Maria, v. 6, n. 1, p. 25-38, jan/ mar. 2013

SILVA, J. P. Análise financeira das empresas. 11ạ Ed. São Paulo: Atlas, 2012. 
SOUZA, A. A. et al. Indicadores de desempenho econômico-financeiro para hospitais: um estudo teórico. Revista de Administração Hospitalar e Inovação em Saúde, Belo Horizonte, v. 3, n. 2, p. 44-55, 2009.

SOUZA, A. A. et al. Indicadores de desempenho para hospitais: análise a partir dos dados divulgados para o público em geral. In: Anais 10 Congresso USP de Controladoria e Contabilidade, São Paulo, 2010.

STRUETT, M. Custeio baseado em atividades em laboratórios de análises clínicas: estudo de caso em um hospital filantrópico. 2005. 165 f. Dissertação (Mestrado em Administração) Universidade Estadual de Londrina, Londrina, 2005.

SULKU, S. N. The health sector reforms and the efficiency of public hospitals in Turkey: provincial markets. European Journal of Public Health, v. 10, p. 1-5, 2011.

VALDMANIS, V. G.; ROSKO, M. D. \& MUTTER, R. L. Hospital Quality, Efficiency, and Inputs Slack Differentials. Health Services Research, v. 43, n. 5, p. 1830-1848, 2008.

YANG, C.; QI, Q. \& HU, H. Analysis of hospital technical efficiency in China: Effect of health insurance reform. China Economic Review, v. 23, p. 865-877, 2012.

VELOSO, G. G. \& MALIK, A. M. Análise do desempenho econômico-financeiro de empresas de saúde. RAE-eletrônica, São Paulo, v. 9, n. 1, art. 2, 2010. 
VENDEMIATTI, M. et al. Conflito na Gestão Hospitalar: O papel da Liderança. Ciência \& Saúde Coletiva, Rio de Janeiro, v. 15 (Supl. 1), p. 1301-1314, 2007.

ZELLER, T. L. et al. A Revised Classification Pattern of Hospital Financial Ratios. Journal of Accounting and Public Policy, v. 15, 199, p. 161-182, 1996. 